\title{
AN INITIAL EXAMINATION FOR VERIFYING SEPARATION ALGORITHMS BY SIMULATION
}

\author{
Allan L. White, NASA Langley, Hampton, Virginia \\ Natasha Neogi, Heber Herencia-Zapana, National Institute of Aerospace, Hampton, Virginia
}

\begin{abstract}
An open question in algorithms for aircraft is what can be validated by simulation where the simulation shows that the probability of undesirable events is below some given level at some confidence level. The problem is including enough realism to be convincing while retaining enough efficiency to run the large number of trials needed for high confidence. The paper first proposes a goal based on the number of flights per year in several regions. The paper examines the probabilistic interpretation of this goal and computes the number of trials needed to establish it at an equivalent confidence level. Since any simulation is likely to consider the algorithms for only one type of event and there are several types of events, the paper examines under what conditions this separate consideration is valid. This paper is an initial effort, and as such, it considers separation maneuvers, which are elementary but include numerous aspects of aircraft behavior. The scenario includes decisions under uncertainty since the position of each aircraft is only known to the other by broadcasting where GPS believes each aircraft to be (ADS-B). Each aircraft operates under feedback control with perturbations. It is shown that a scenario three or four orders of magnitude more complex is feasible. The question of what can be validated by simulation remains open, but there is reason to be optimistic
\end{abstract}

\section{Introduction}

This paper first considers the number of trials needed in a Monte Carlo simulation to show some algorithm or procedure is effective enough to satisfy the proposed requirement. Demonstrating how to compute the number of trials proceeds by example.

Following is an outline of this paper's major sections:

- A discussion of the scenario that describes the aircraft, the flight space, the feedback control with perturbations, and the model for GPS errors
- A presentation of orientation graphs showing that under the assumptions in the previous section, the minimum-distance point determines the relative angles of the aircraft and the results are presented in two orientation graphs that show when the flight paths are collinear and when they are opposing. These orientation graphs are used to choose the flight paths in a random manner.

- A description of flight path selection, placement, and GPS data collection used for deciding aircraft maneuvers

- A presentation of the decision algorithms and the two maneuvers of weave for collinear flight paths and jog for opposing flight paths.

- A discussion of related work followed and proposed future work

This current work demonstrates that a separation algorithm with decisions under uncertainty and with aircraft perturbations can be shown to meet a rigorous goal at a correspondingly high confidence level. It is also shown that this project can accept a three to four magnitude increase in complexity and still remain viable, but clearly this is not enough of a margin to include every detail in a global setting. Future work is needed in sensitivity analysis to determine what must be included in the simulation.

\section{Probability and Statistics}

\section{Choosing the Requirement}

First, loss-of-separation is not catastrophic, and it is not likely to be subjected to the high standard used in this paper. Separation maneuvers were chosen because they are a convenient place to begin the study of algorithms and they can incorporate numerous features of aircraft behavior. In addition, loss-of-separation is the first step leading to a collision, which is catastrophic.

We must choose a quantitative goal, and it must be related to air traffic. There are about 10 million 
flights per year in the continental United States and about the same number in Europe [12, 13]. We will be conservative and anticipate future increases by assuming 15 million flights a year in an air space.

For this paper, an event is the occurrence of certain undesirable items, and loss-of-separation is one type of event, but there may be other types of events. The hypothetical goal is no more than one event per year.

\section{Probability}

As it stands, the goal stated above is not a probability statement, and it requires interpretation.

For simplicity, the typical assumption is that all flights are equivalent and independent, and the typical interpretation of the goal is that the expected number of events for 15 million flights be equal to one. Using the expectation does not require any more information, but it does have a disadvantage as will be seen below.

The disadvantage of this interpretation appears when we consider the probability of more than one event during 15 million flights. It's reasonable to want the probability of more than one event to be low, but it will be shown that using the expectationinterpretation does not guarantee this. On the other hand, the low-probability approach raises the question of how low we wish the probability to be. Setting the expectation equal to 1 and assuming that the flights are equivalent and independent, the distribution is binomial with the probability of an event equal to $6.6667 \mathrm{e}-8$ per flight. The binomial distribution with parameter $\mathrm{p}$ gives the probability of zero or one event during 15 million flights as

$$
\begin{aligned}
Q & =(1-p)^{15000000}+15000000 p(1-p)^{14999999} \\
& =0.7358 \text { if } p=6.6667 e-8
\end{aligned}
$$

The probability of two or more events for $\mathrm{p}=$ $1 \mathrm{e}-7$ is $1-\mathrm{Q}=0.2642$. Hence, if the probability of an event is based on the expectation, then the probability of more than one event during 15 million flights is greater than $25 \%$.

We will adopt the more stringent requirement that there is less than a $10 \%$ chance of more than one event per year. Setting $Q=0.1$ in formula (1) and solving gives $\mathrm{p}=3.5 \mathrm{e}-8$.

\section{Multiple Events and Lack of Information}

A problem in establishing that a loss-ofseparation algorithm meets a goal is that loss of separation is one event among many. Hence, showing that the probability of loss of separation during a flight is less than $3.5 \mathrm{e}-8$ may not be sufficient since there are other events and their probabilities accumulate. The problem is compounded since when studying types of events, especially the prevention of events, it is useful to distinguish between the potential for an event and the event itself. For instance, two aircraft on a collision course is a potential for an event, but successful maneuvering will result in no event. In addition, there may be multiple causes for an event or an event may require multiple causes. There may be no cause for alarm if two aircraft are on a collision course unless some malfunction prevents successful maneuvering. Hence, a precise probability analysis for loss-of-separation requires an encyclopedic knowledge of events and their causes which the authors, at least, do not currently posses. Nevertheless, an elementary, incomplete analysis can offer some guidance. One approach in the absence of information is to be conservative in the modeling and use probabilities that overestimate the likelihood.

We begin with a simplified scenario and then generalize it. Suppose there are K types of events. Let $C_{i}$ be the set of causes for event $A_{i}$. Let $B$ (for benign) be the set where no causes for an event occur. The initial simplifying assumption is that the $\mathrm{C}_{\mathrm{i}}$ and $\mathrm{B}$ partition the set of flight conditions. That is, the intersection of two different sets is empty, and their union is the entire set. This initial simplifying assumption is justified if events are rare and flights with more than one event are rare enough to be ignored. With this approach, the study of an event $\mathrm{i}$ consists of the study of the effect of the set $\mathrm{C}_{\mathrm{i}}$. For instance, for this study of loss-of-separation, the causes are deviations from the flight paths due to feedback control and external perturbations. The realism of the simulation is increased by adding more causes.

Let $\mathrm{P}\left(\mathrm{A}_{\mathrm{i}} \mid \mathrm{C}_{\mathrm{i}}\right)$ be the conditional probability of an event given that its causes appear. Then we want

$$
\mathrm{P}\left(\mathrm{A}_{1} \mid \mathrm{C}_{1}\right) \mathrm{P}\left(\mathrm{C}_{1}\right)+\mathrm{P}\left(\mathrm{A}_{2} \mid \mathrm{C}_{2}\right) \mathrm{P}\left(\mathrm{C}_{2}\right)+\ldots
$$




$$
+\mathrm{P}\left(\mathrm{A}_{\mathrm{K}} \mid \mathrm{C}_{\mathrm{K}}\right) \mathrm{P}\left(\mathrm{C}_{\mathrm{K}}\right) \leq \mathrm{p} .
$$

Based on the assumption that there is a positive probability that a flight is routine (no cause for an event appears), we have

$$
\mathrm{P}\left(\mathrm{C}_{1}\right)+\ldots+\mathrm{P}\left(\mathrm{C}_{\mathrm{K}}\right)<1 .
$$

Using this assumption, one way to accomplish this is to have $P\left(A_{i} \mid C_{i}\right) \leq p$ for all $i$ since this gives

$$
\begin{aligned}
& \mathrm{P}\left(\mathrm{A}_{1} \mid \mathrm{C}_{1}\right) \mathrm{P}\left(\mathrm{C}_{1}\right)+\mathrm{P}\left(\mathrm{A}_{2} \mid \mathrm{C}_{2}\right) \mathrm{P}\left(\mathrm{C}_{2}\right)+\ldots \\
& \quad+\mathrm{P}\left(\mathrm{A}_{\mathrm{K}} \mid \mathrm{C}_{\mathrm{K}}\right) \mathrm{P}\left(\mathrm{C}_{\mathrm{K}}\right) \\
& \quad \leq \mathrm{pP}\left(\mathrm{C}_{1}\right)+\mathrm{pP}\left(\mathrm{C}_{2}\right)+\ldots+\mathrm{p} \mathrm{P}\left(\mathrm{C}_{\mathrm{K}}\right) \\
& \quad \leq \mathrm{p}\left[\mathrm{P}\left(\mathrm{C}_{1}\right)+\ldots+\mathrm{P}\left(\mathrm{C}_{\mathrm{K}}\right)\right] \leq \mathrm{p} .
\end{aligned}
$$

The generalization of the above eliminates the partition requirement. That is, different $\mathrm{C}_{\mathrm{i}}$ can have a non-empty intersection, allowing for more than one event per flight. The reasoning above still holds if $\mathrm{P}\left(\mathrm{C}_{1}\right)+\ldots+\mathrm{P}\left(\mathrm{C}_{\mathrm{K}}\right) \leq 1$, which this paper will assume.

There are two cases where the approach above requires modification. First, if the sets $\mathrm{C}_{i}$ have significant overlap, then the probabilities can sum to greater than 1. If a bound for the sum of probabilities is known and it is less than $\mathrm{M}$, then it is sufficient to demonstrate $\mathrm{P}\left(\mathrm{A}_{\mathrm{i}} \mid \mathrm{C}_{\mathrm{i}}\right) \leq \mathrm{q}$ where $\mathrm{q} \mathrm{M} \leq 1$, although if there is significant overlap, then the studies will have to examine the probability that a single set of causes produces several events.

Second, a scenario that would require a different type of analysis is if a set of causes had a high probability of producing an event. That is, for some $\mathrm{j}, \mathrm{P}\left(\mathrm{A}_{\mathrm{j}} \mid \mathrm{C}_{\mathrm{j}}\right)$ cannot be made small. In this case, the alternative is to arrange things so that $\mathrm{C}_{\mathrm{j}}$ is small.

\section{Confidence Levels and Number of Trials}

The driver for Monte Carlo is the required confidence level which is a quantitative statement about the quality of the experiment. The frequency interpretation is that a confidence level of $100(1-\mathrm{h}) \%$ means there is a $100 \mathrm{~h} \%$ or less chance that the experiment has misled us. This paper takes the point of view that the quality of the experiment should match the quality of the desired results. That is, if the probability to be established is $\mathrm{p}$, then the confidence level should be at least $100(1-\mathrm{p}) \%$. Hence, this paper will seek confidence levels of at least 100(11e-7)\%. The confidence level may need to be even higher because loss-of-separation is only one event among many. The final confidence level must combine the confidence level of a number of experiments. A result in combining confidence levels is the following.

Theorem: Suppose $\left(a_{j}, b_{j}\right)$ is a $100\left(1-h_{j}\right) \%$ confidence interval for $\theta_{j}$ for $1 \leq \mathrm{j} \leq \mathrm{n}$, then $\left[\left(a_{1}, b_{1}\right), \ldots\left(a_{n}, b_{n}\right)\right]$ is a $100\left(1-h_{1}-\ldots-h_{n}\right) \%$ confidence interval for $\left(\theta_{1}, \ldots, \theta_{n}\right)$.

For example, if there are 10 parameters to be estimated with a desired overall confidence level of $100(1-1 \mathrm{e}-7) \%$, then it is sufficient to estimate each of the parameters at the $100(1-1 \mathrm{e}-8) \%$ level. In general, the individual confidence intervals do not need to be the same although the lack of confidence must have a sum less than or equal to $1 \mathrm{e}-7$. Assuming that all the trials are successful and given a desired probability $\mathrm{p}$ and confidence level $\mathrm{h}$, the formula for computing the number $\mathrm{n}$ of trials is

$$
(1-p)^{n}=h \text {. }
$$

The reasoning is that (1-p) is the probability of success (equivalently the non-occurrence of a failure) and repeated successes ( $\mathrm{n}$ of them) imply that $\mathrm{p}$ is small.

For this paper, we assume there are 100 types of events. Hence using $\mathrm{p}=3.5 \mathrm{e}-8$ and $\mathrm{h}=3.5 \mathrm{e}-10$ in formula (5) gives $n=620,000,000$.

\section{Feasibility}

For the scenario described below, the 620 million trials took 9 days on a desktop computer using an interpretive language.

One can use a compiled language for an order of magnitude gain in efficiency and run the program for 100 days, which permits two orders of magnitude increase in the complexity of the simulation. 
Monte Carlo trials differ by their choices of random numbers which implies that different computers can run different trials. One thousand computers cost less than two man-years, which permits another three orders of magnitude increase in complexity.

\section{Scenario}

\section{The Aircraft}

This study considers two aircraft. The aircraft neither change altitude nor speed. The speeds are between 0.20 kilometers per second and 0.25 kilometers per second which is equivalent to speeds between 389 and 486 knots.

If the flight paths are collinear, the slower craft performs a weave for delay. If the paths are opposing, the slower craft performs a jog behind the faster craft.

During any maneuver, the flight path changes are limited to 1 degree per second which is within the range of all commercial airliners.

\section{Flight Path Error}

The aircraft maneuver under feedback with perturbation according to

$$
\mathrm{a}(\mathrm{k})=-0.5[\mathrm{~s}(\mathrm{k})-\mathrm{sd}(\mathrm{k})]-0.5[\mathrm{v}(\mathrm{k})-\mathrm{vd}(\mathrm{k})]+\mathrm{u}(\mathrm{k})
$$

where

$\mathrm{a}(\mathrm{k})$ is the acceleration from $\mathrm{k}$ to $\mathrm{k}+1$

$\mathrm{s}(\mathrm{k})$ is the position at $\mathrm{k}$

$\mathrm{sd}(\mathrm{k})$ is the desired position at $\mathrm{k}$

$\mathrm{v}(\mathrm{k})$ is the velocity at $\mathrm{k}$

$\mathrm{vd}(\mathrm{k})$ is the desired velocity from $\mathrm{k}$ to $\mathrm{k}+1$

$\mathrm{u}(\mathrm{k})$ is the perturbation

The perturbation is a Markov-Gaussian process where $u(k+1)$ is a normal distribution with mean $0.1 \mathrm{u}(\mathrm{k})$ and standard deviation of 0.01 . This perturbation includes errors from navigation, feedback control, and wind gusts.

\section{GPS Error}

It is assumed that there is a $95 \%$ chance that the GPS reading is within 10 meters of the actual position [9]. Assuming the $\mathrm{x}$ and $\mathrm{y}$ coordinates are independent and normal, this condition is satisfied if $\mathrm{x}$ and $\mathrm{y}$ have a standard deviation of 4.1 meters.

During the first 100 seconds of the simulation, each aircraft sends the GPS reading of its position, and the decisions are based on these GPS signals.

\section{GPS Usage}

For clarity, we mention that GPS data is used in two different ways in this scenario. One is implicit: it is part of aircraft navigation and feedback control, and it is included as part of the Markov-Gaussian flight perturbation.

The second is explicit. Once a second, the GPS receiver on an aircraft reads in the signals and produces a GPS estimate of position. This estimate is broadcast to other aircraft without any additional processing.

\section{Causes for an Event in this Simulation}

From the above, it is clear there are four factors in creating a loss-of-separation event in this simulation. First, the aircraft are on courses that bring them close. Second, GPS error may cause the aircraft to not maneuver when they should. Third, an algorithm may make an incorrect decision. Fourth, even if GPS is accurate enough and the algorithm makes the correct decision, flight perturbations may cause lack of separation.

In the notation of the second section, these four factors form the causes $C_{i}$, and the event $A_{i}$ is a loss of separation. The goal of the simulation is to show that $\mathrm{P}\left(\mathrm{A}_{\mathrm{i}} \mid \mathrm{C}_{\mathrm{i}}\right)$ is small.

\section{The Orientation Graphs}

\section{Preliminaries}

The orientation graphs summarize the possible angles of approach for the two aircraft. They give the conditions under which the flight paths are collinear or opposing, and they are used to randomly choose the flight paths during the simulation.

Assume two aircraft are at the same altitude and both have a straight line as their flight paths. We show that the points at which the aircraft are closest together (the minimum points) and the relative speed of the aircraft determines their angles of approach. If 
the speeds differ, then there are two angles of approach for each pair of minimum points.

For algebraic convenience, let the first aircraft travel along the $\mathrm{x}$-axis and let the aircraft be a minimum distance apart when the first aircraft is at the point $(0,0)$.

Let

$\mathrm{v}=$ speed of first aircraft

$\mathrm{cv}=$ speed of second aircraft where $0<\mathrm{c} \leq 1$

$(a, b)=$ minimum point for the second aircraft

$\alpha=$ flight angle of the second aircraft

This scenario is depicted in figure 1.

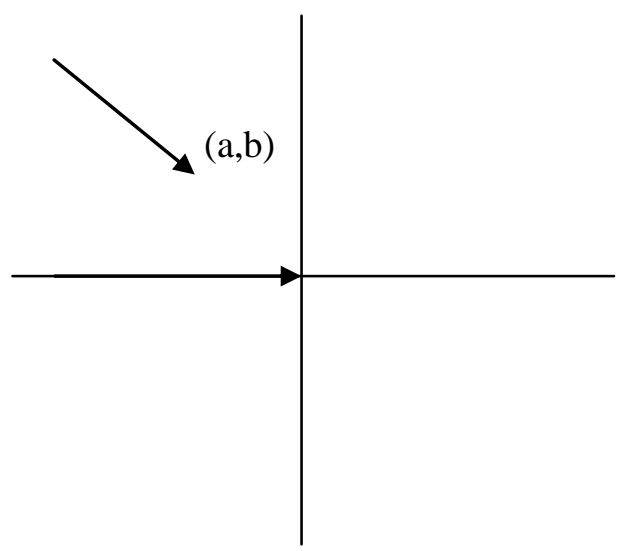

Figure 1. Flight paths and the Minimum Points of $(0,0)$ and $(a, b)$ for Two Aircraft

Assume the aircraft are a minimum distance apart when time $\mathrm{t}=0$. This implies that time can have negative values in the parametric equations that describe their flight paths.

The parametric equations are

$$
\begin{aligned}
& x_{1}=t v \\
& y_{1}=0 \\
& x_{2}=a+t c v \cos \propto \\
& y_{2}=b+t c v \sin \propto
\end{aligned}
$$

The square of the distance and its first two derivatives are

$$
\begin{gathered}
s^{2}=[a+t c v \cos \propto-t v]^{2} \\
+[b+t c v \sin \propto]^{2} \\
\begin{array}{c}
\frac{d\left(s^{2}\right)}{d t}=2[a+t c v \cos \propto-t v][c v \cos \propto-v] \\
+2[b+t c v \sin \propto][c v \sin \propto] \\
\frac{d^{2}\left(s^{2}\right)}{d t^{2}}=2[c v \cos \propto-v]^{2}+2[c v \sin \propto]^{2}
\end{array}
\end{gathered}
$$

By the second derivative, the function has a minimum when the first derivative is zero.

\section{The Solution Pairs}

Set the first derivative and $t$ equal to zero and solve to get

$$
\cos \propto=\frac{a^{2} \pm b \sqrt{\left(a^{2}+b^{2}\right) c^{2}-a^{2}}}{c\left(a^{2}+b^{2}\right)}
$$

Substitution gives the solution pairs

$$
\cos \propto=\frac{a^{2}+b \sqrt{\left(a^{2}+b^{2}\right) c^{2}-a^{2}}}{c\left(a^{2}+b^{2}\right)}
$$

$$
\sin \propto=\frac{a b-a \sqrt{\left(a^{2}+b^{2}\right) c^{2}-a^{2}}}{c\left(a^{2}+b^{2}\right)}
$$

and

$$
\cos \propto=\frac{a^{2}-b \sqrt{\left(a^{2}+b^{2}\right) c^{2}-a^{2}}}{c\left(a^{2}+b^{2}\right)}
$$

$$
\sin \propto=\frac{a b+a \sqrt{\left(a^{2}+b^{2}\right) c^{2}-a^{2}}}{c\left(a^{2}+b^{2}\right)}
$$




\section{The No-Fly Zone}

If $\mathrm{c}=1$, the discriminant is non-negative. Suppose $0<\mathrm{c}<1$. Setting

$\left(a^{2}+b^{2}\right) c^{2}-a^{2} \geq 0$

gives

$$
\begin{aligned}
& b^{2} \frac{c^{2}}{1-c^{2}} \geq a^{2} \\
& |b| \geq \sqrt{\frac{1-c^{2}}{c^{2}}}|a|
\end{aligned}
$$

Hence, if the second aircraft is slower, there are no minimum points in the shaded regions in figure 2 and 3 where the equations for the lines bounding the shaded regions are

$$
b=\sqrt{\frac{1-c^{2}}{c^{2}}} a
$$

and

$$
b=-\sqrt{\frac{1-c^{2}}{c^{2}}} a
$$

\section{Examination of the Cosine for the First pair}

The important parameter is the sign of the cosine of $\alpha$ since if it is positive the aircraft are flying coincident courses while if it is negative the aircraft are flying opposing courses.

Consider the positive and negative regions for

$$
\cos \propto=\frac{a^{2}+b \sqrt{\left(a^{2}+b^{2}\right) c^{2}-a^{2}}}{c\left(a^{2}+b^{2}\right)}
$$

The expression above is positive if $\mathrm{b} \geq 0$.

Suppose $b<0$. Since the denominator is positive, alternately set the numerator

$$
a^{2}+b \sqrt{\left(a^{2}+b^{2}\right) c^{2}-a^{2}}
$$

greater than zero and less than zero to get

$$
\cos \alpha>0 \text { if }|b| \leq \frac{|a|}{c}
$$

$$
\cos \alpha<0 \text { if }|b| \geq \frac{|a|}{c}
$$

Note that $\frac{|a|}{c}$ is greater in magnitude than $\sqrt{\frac{1-c^{2}}{c^{2}}}|a|$.

\section{Examination of the Sine for the First Pair}

Next consider the positive and negative regions for the associated

$$
\sin \propto=\frac{a b-a \sqrt{\left(a^{2}+b^{2}\right) c^{2}-a^{2}}}{c\left(a^{2}+b^{2}\right)}
$$

It is sufficient to examine the numerator.

Suppose $a>0$ and $b>0$. Set

$$
a b-a \sqrt{\left(a^{2}+b^{2}\right) c^{2}-a^{2}} \geq 0
$$

Cancel the a, place the radical on the right-hand-side and square to get

$$
a^{2}+b^{2} \geq\left(a^{2}+b^{2}\right) c^{2}
$$

which is always true. positive.

Suppose $\mathrm{a}<0$ and $\mathrm{b}<0$. Then the numerator is

Suppose $\mathrm{a}<0$ and $\mathrm{b}>0$. Set

$$
a b-a \sqrt{\left(a^{2}+b^{2}\right) c^{2}-a^{2}} \leq 0
$$

Placing the term with the radical on the rightand-side, dividing by a $<0$, and squaring gives

$$
a^{2}+b^{2} \geq\left(a^{2}+b^{2}\right) c^{2}
$$


which is always true.

Suppose $\mathrm{a}>0$ and $\mathrm{b}<0$. Then the numerator is negative.

\section{The Regions for the First Pair}

For the solution pair

$$
\begin{aligned}
& \cos \propto=\frac{a^{2}+b \sqrt{\left(a^{2}+b^{2}\right) c^{2}-a^{2}}}{c\left(a^{2}+b^{2}\right)} \\
& \sin \propto=\frac{a b-a \sqrt{\left(a^{2}+b^{2}\right) c^{2}-a^{2}}}{c\left(a^{2}+b^{2}\right)}
\end{aligned}
$$

the orientation graph for (cosine, sine) is given in figure 2 .

For the shaded area in figure 2 (and figure 3), the equations for the no-fly zone are

$$
b=\sqrt{\frac{1-c^{2}}{c^{2}}} a \text { and } b=-\sqrt{\frac{1-c^{2}}{c^{2}}} a
$$

while the equations for the lines in quadrants three and four (or quadrants one and two) are

$$
b= \pm \frac{a}{c} \text {. }
$$

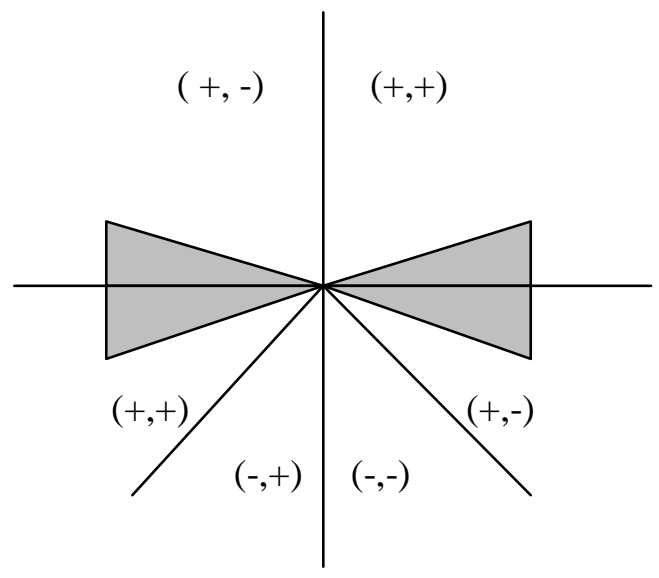

Figure 2. Orientation Graph for the First Solution Pair

\section{The Regions for the Second Pair}

Proceeding similarly, the orientation graph for the second the solution pair

$$
\begin{aligned}
& \cos \propto=\frac{a^{2}-b \sqrt{\left(a^{2}+b^{2}\right) c^{2}-a^{2}}}{c\left(a^{2}+b^{2}\right)} \\
& \sin \propto=\frac{a b+a \sqrt{\left(a^{2}+b^{2}\right) c^{2}-a^{2}}}{c\left(a^{2}+b^{2}\right)}
\end{aligned}
$$

is given in figure 3 .

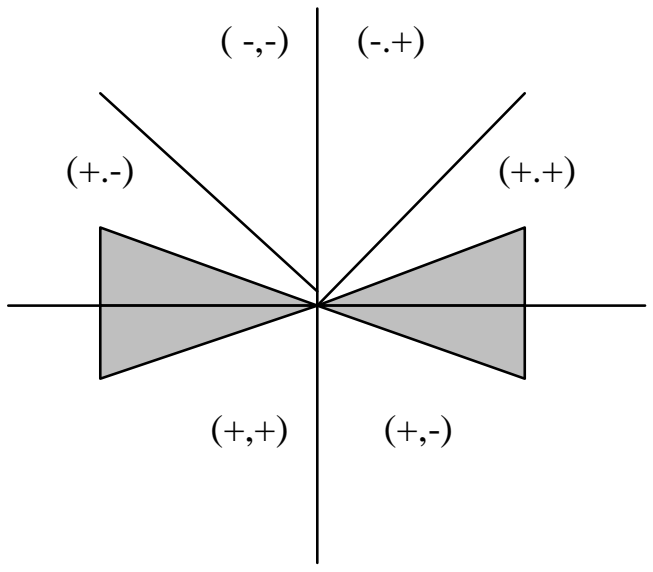

Figure 3. Orientation Graph for the Second Solution Pair

\section{Distance Restriction}

There is one additional restriction for collinear paths. To keep the distances considered from becoming very large and to give the aircraft time to make a decision and maneuver, nearly parallel flight paths that are close together are excluded from this simulation, as they probably are in actual airspace.

The exclusion zone for the minimum point of the slower aircraft is between the angles of $5 \pi / 6$ and $7 \pi / 6$. 


\section{Initializing the flight Paths}

Randomly, with equal probability, choose which orientation graph to use. Since opposing aircraft are likely to be at different altitudes, choose collinear with probability 0.9 and opposing with probability 0.1 .

Pick a minimum point for the second aircraft uniformly from the appropriate set of points within $10 \mathrm{~km}$ of the origin. Compute the cosine and sine.

Choose the two velocities independently from the uniform distribution on $[0.20,0.25]$. and assign the higher velocity to the aircraft moving on the $\mathrm{x}$ axis. Use the minimum points, the angles, and the velocities to compute the starting points at $\mathrm{t}=-500$ seconds.

For generality, rotate the axes with an angle chosen uniformly from $[0,2 \pi]$, with a $50 \%$ probability, reflect the axes with an angle chosen uniformly from $[0, \pi]$, and independently translate the $\mathrm{x}$ an $\mathrm{y}$ axis with the quantities chosen from the uniform distribution on $[-100,100]$.

Finally, both aircraft operate under feedback control with perturbations for 100 seconds. The GPS position (with error) is broadcast once a second during this period. This GPS broadcast is the basis for the decision procedures.

\section{Decision Procedures}

\section{Outline}

This section describes the methods used to examine the GPS estimates, decide if a maneuver is necessary, and determine which aircraft should maneuver and how it should maneuver.

The decision procedure contains six steps which are performed $n$ order: (1) estimate the speeds, (2) estimated the cosine and sine of the flight paths, (3) estimating the starting points of the two flight paths, (4) form the parametric equations for the flight paths using the estimated speeds and angles, and take the derivative of the distance between the aircraft to decide if the minimum distance is less than the required separation, (5) if a maneuver is required, decide if the paths are collinear or approaching, and (6) if a maneuver is required, decide if the slower aircraft is to the left or right of the faster aircraft.

\section{Estimating the Speed and Flight Angles}

The successive GPS readings are used to estimate the speed for each one second interval, and these are averaged for the final estimate.

Similarly, the GPS readings are used to estimate cosine and sine for each one second interval, and these are averaged for the final estimate.

\section{Estimating the Starting Points of the Flight Paths}

Let $\alpha$ be the estimated flight angle, let $\mathrm{v}$ be the estimated velocity, and let $x_{k}$ be the GPS reading of the $\mathrm{x}$ coordinate at time $\mathrm{k}$. Let

$$
\mathrm{Sx}_{\mathrm{k}}=\mathrm{x}_{\mathrm{k}}-(\mathrm{k}-1) \mathrm{v} \cos \alpha
$$

for $\mathrm{k}=1$ to 100 . Average the $\mathrm{sx}_{\mathrm{k}}$ for the estimate of the $\mathrm{x}$ coordinate of the starting of the starting point. Similarly for the y coordinate.

\section{Collinear or Approaching}

In figure 4 below, the coordinate axes have been translated (in the $\mathrm{x}$ and $\mathrm{y}$ direction) to place the origin at the intersection of the flight paths. This translation preserves angles. The flight paths for the aircraft are denoted by the solid vectors labeled 1 and 2 , and the continuations of the flight paths are given by the dotted lines. Consider either $\cos (\varphi-\psi)$ or $\cos (\psi-$ $\varphi)$. If the cosine is positive, then the difference of the angles is between $-\pi / 2$ and $\pi / 2$, and the aircraft are collinear. If the cosine is negative, then the difference of the angles is between $\pi / 2$ and $3 \pi / 2$, and the aircraft are approaching.

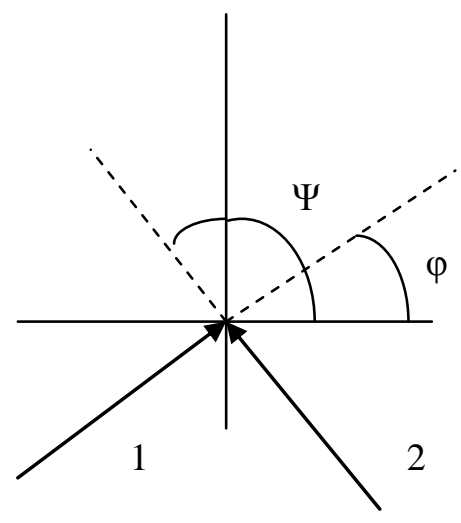

Figure 4. Determining if Collinear or Opposing 


\section{Left or Right}

For the collinear case, in figure 5 below, the coordinate axes have undergone an angle-preserving translation to place the intersection of the flight paths at the origin. The flight paths are denoted by the solid vectors labeled 1 and 2, and their continuations are given by the dotted lines. If $\sin (\psi-\varphi)$ is positive, then plane 1 is to the left of plane 2 . Similarly, $\sin (\varphi-\psi)$ negative indicates aircraft 2 is to the right of aircraft 1 .

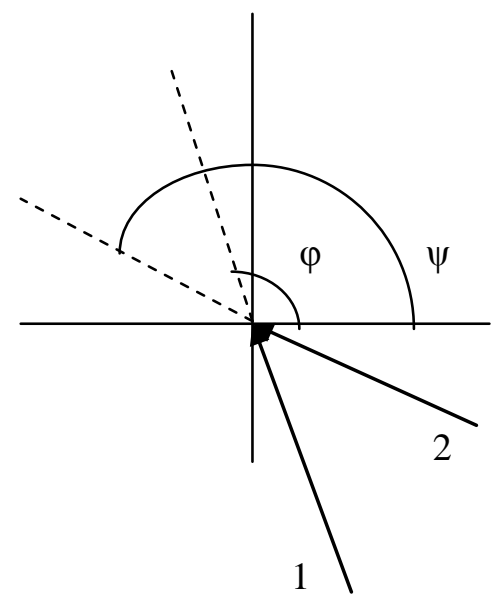

Figure 5. Determining Left or Right for Collinear Paths

Using the same procedure and notation for approaching aircraft in figure 6 , the difference between the angles lies between $\pi / 2$ and $3 \pi / 2$. Since $\pi / 2 \leq \psi-\varphi<\pi$, the sine of the difference is positive, indicating aircraft 2 is to the right of aircraft 1 . Since $\pi<\varphi-\psi \leq 3 \pi / 2$, the sine of the difference is negative, indicating aircraft 1 is to the left of aircraft 2.

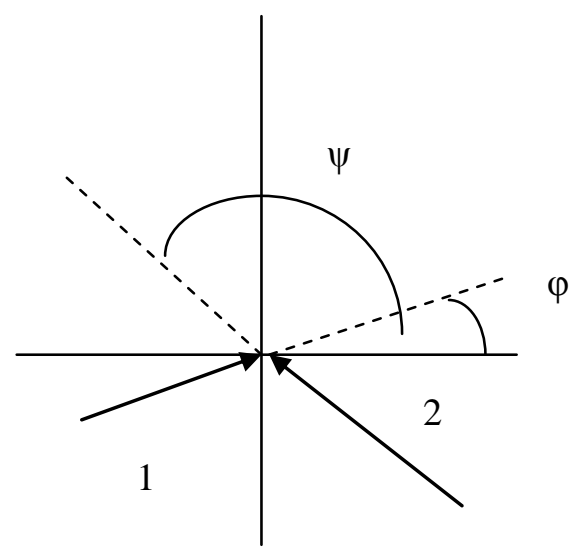

Figure 6. Determining Left or Right for Opposing Paths

\section{The Separation Maneuvers}

As described above, the aircraft are placed at their starting points and GPS readings are recorded for the first 100 seconds whereupon it is decided which aircraft should maneuver and the respective desired flight paths are computed. The aircraft follow these paths, using feedback with perturbations as presented in section three, for 900 more seconds. During this time, the distance is computed at each second to check that the aircraft maintain a distance of at least 10 kilometers.

The flight paths for a weave and a jog are shown in figures 7 and 8 . For each, the original flight path begins at $(0,0)$ and is horizontal. The increasing (blue) line is the $\mathrm{x}$ coordinate, and the rising and falling (green) line is the y coordinate.

In figures 7 and 8 , the horizontal units are seconds and the vertical units are kilometers. These graphs do not plot $\mathrm{y}$ against $\mathrm{x}$, but $\mathrm{x}$ and $\mathrm{y}$ parametrically as a function of time. 


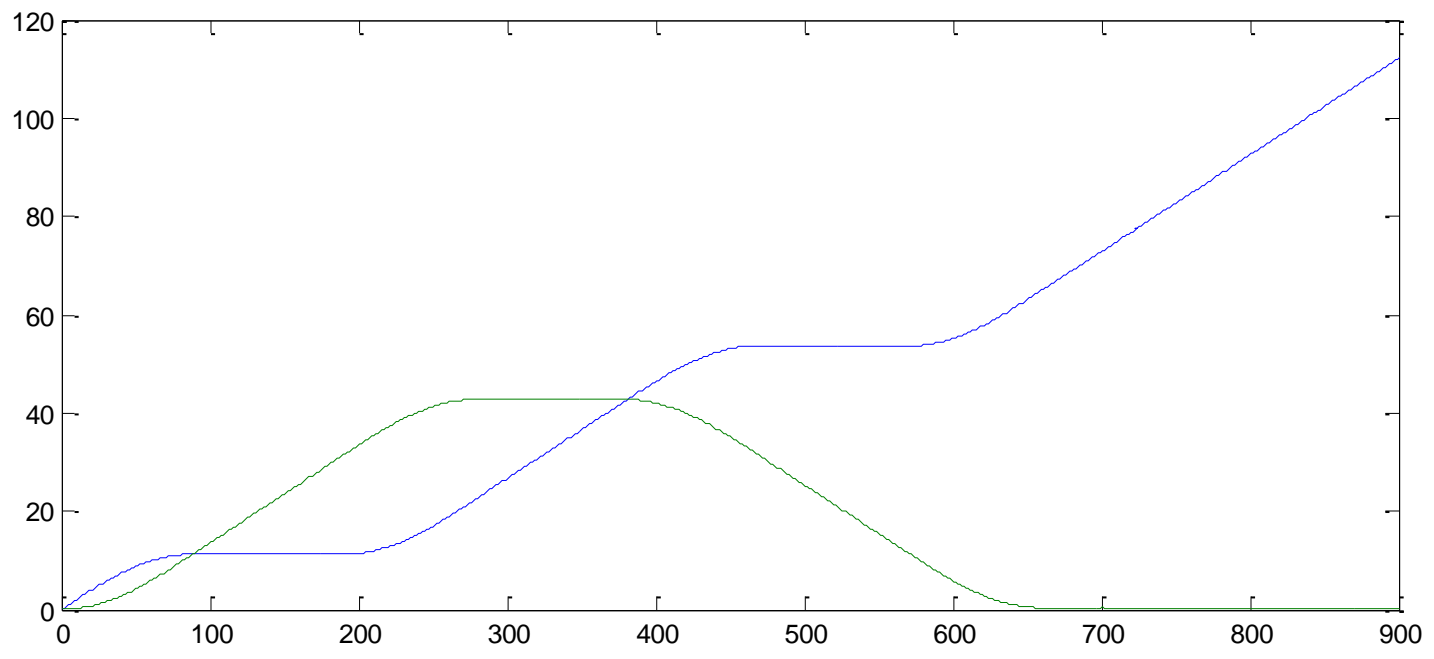

Figure 7. A Weave to the Left for the Slower Aircraft on a Collinear path

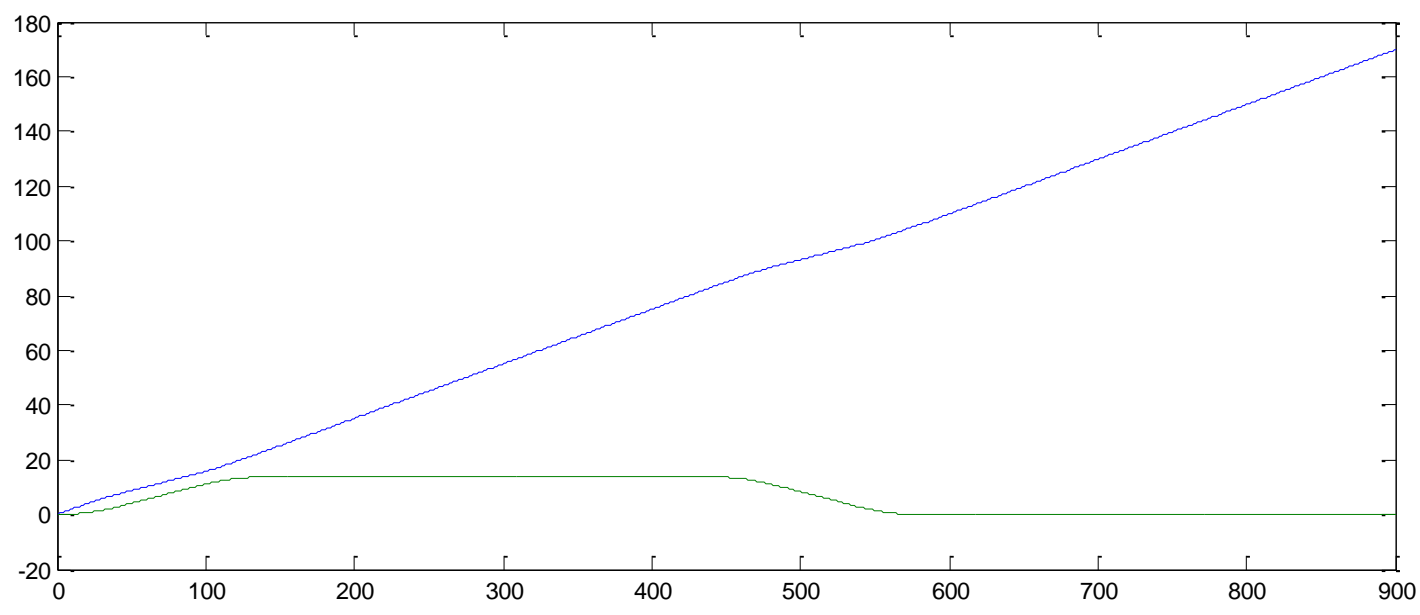

Figure 8. A Jog to the Left for the Slower Aircraft on an Opposing Path

\section{Further Work}

We need a description of GPS error as a stochastic process. Failing this, we can use a worstcase approach: there is general agreement that GPS performs better than the chosen stochastic process. Alternately, there is performing a sensitivity analysis to determine how much error a decision algorithm can tolerate.
It is also desirable to have a stochastic process that describes the worst case flight perturbations of a commercial airliner. This lets a simulation be performed for all aircraft, and it eliminates the need to include flight dynamics and feedback control in the simulation.

From the point of view of the above two items, the error distribution used for GPS and the feedback 
and perturbations used for flight paths in this paper can be considered place holders - replacing them with the stochastic processes for GPS and aircraft will not significantly increase the computational burden.

One possibility when using worst-case processes for GPS and flight path is that, under some circumstances, the algorithm cannot decide with high confidence which aircraft should maneuver. In this case, it is necessary to have algorithms that let both aircraft maneuver without maneuvering into each other.

This paper did not consider false alarms where an aircraft performed an unnecessary maneuver.

There is a need for maneuvers that change altitude in case the airspace at a certain altitude is crowded.

We need to consider more flexible approaches to receiving GPS signals and we need to consider less benign behavior of the GPS transponder, including equipment failure. Along with this, we need algorithms that can handle more flexible behavior and degraded performance.

\section{Discussion for References}

There is no lack of publications about separation assurance or, when things get more desperate, collision avoidance. In comparing this effort to other publications, the salient points are (1) examination of the regulatory requirements as probability conditions, (2) integrating the combined realism of feedback control and perturbations, and (3) determining and performing the necessary Monte Carlo trials to establish a high confidence level.

The paper [1] sets up a framework to review 68 conflict detection and resolution methods. The paper [2] examines a three dimensional algorithm for collision avoidance. The paper [3] includes human in the loop with the intent of moving from sector to global implications to improve efficiency. The paper [4] identifies possible areas to investigate for improvement of efficiency,. The paper [5] proposed a conflict resolution algorithm and tested it using real data. The paper [6] develops and tests an operating concept and a laboratory analysis methodology to examine how four-dimensional trajectory analysis methods could support higher levels of automation for separation assurance while recognizing that trajectory prediction uncertainties can be on the same order of magnitude as safe separation criteria, which implies that uncertainty is an important consideration. The paper [7] attempts a global simulation of the Australian airspace to study the feasibility of free flight, and it contains numerous realistic elements. The paper [8] applies integer programming to solve conflict resolution for multiple aircraft, and several examples are presented. The paper [9] contains information on GPS error, and the paper [10] is an initial examination of worst case flight perturbations. The paper [11] considers distributed-versuscentralized control and optimization of aircraft maneuvers.

\section{Summary}

This paper describes a work in progress where the open question is whether or not it is possible to establish algorithms for aircraft by simulation. The challenge is including enough detail to be realistic while remaining efficient enough to run the number of trials necessary to reach a high confidence level. This paper was an initial examination of maintaining separation in the presence of uncertainty - GPS error and flight perturbations.

\section{References}

[1] J. Kuchar and L. Yang, A Review of Conflict Detection and Resolution Modeling Methods, IEEE Transactions on Intelligent Systems, Vol. 1, No. 4, December 2000, pp. 179-189.

[2] M. Christodoulou and S. Kodazakis, Automatic Commercial Aircraft-Collision Avoidance in Free Flight: The Three Dimensional Problem, IEEE Transactions on Intelligent Systems, Vol. 7, No. 2, June 2006, pp. 242-249.

[3] B. Sullivan and S. Malsam, Development of a Real-Time Virtual Airspace Simulation Capability for Air Traffic Management Research, Proceedings of AIAA Modeling and Simulation Technologies Conference and Exhibit 5-8 August, 2002, Monterey. 
[4] D. Sweet, D. Manikonda, V. Aronson, R. Roth, and M. Blake, Fast-Time simulation System for Analysis of Advanced Air Transportation Concepts, Proceedings of AIAA Modeling and Simulation Technologies Conference and Exhibit, 5-8 August 2002, Monterey, California.

[5] T. Farley and H. Erzberger, Fast-Time Simulation Evaluation of a Conflict Resolution Algorithm Under High Air Traffic Demand, Proceedings of

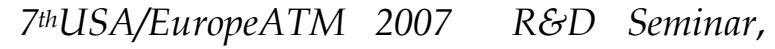
Barcelona, Spain, 2-5 July 2007.

[6] D. McNally and C. Gong, Concept and Laboratory Analysis of Trajectory-Based Automation for Separation Assurance, Air Traffic Control Quarterly, Vol. 15(1), pp. 3563.

[7] S. Alam and H. Abbass, ATOMS: Air Traffic Operations and Management Simulator, IEEE Transactions Intelligent Systems, Vol. 9, No. 2, June 2008, pp. 209-225.

[8] L. Pallottino, E. Feron, and A. Bicchi, Conflict Resolution Problems for Air Traffic Management Systems Solve With Mixed Integer Programming, , IEEE Transactions on Intelligent Systems, Vol. 3, No. 1, March 2002, pp. 3-11
[9] S. Mohleja and G Wang, Modeling ADS-B Position and Velocity Errors for Airborne Merging and Spacing in Interval Management Applications, Center for Advanced Systems Development (CASD), The MITRE Corporation, Case \#10-3026

[10] E. Crisostoni, A. Lecchini-Visintini, and J. Maciejowski, Combining Monte Carlo and Worst-Case Methods for Trajectory Predictions in Air Traffic Control: A Case Study, <www.control.eng.cam.ac.uk/al394/ACA09.pdf>.

[11] K. Bilimoria, B. Sridhar, G. Chatterji, K. Sheth, and S. Grabbe, FACET: Future ATM Concepts Evaluation Tool, Third USA/Europe Air-Traffic management R\&D Seminar, Napoli, Italy, 13-16 June 2000.

[12] Research and Innovative Technology Administration, Bureau of Transportation Statistics, "U. S. Flight Statistics," Web. 14 August 2012. <www.transtats.bts.gov>.

[13] The European Organization for the Safety of Air Navigation, "Statistics and Forecasts," Web. June 2012. < www.eurocontrol.inc>.

31 st Digital Avionics Systems Conference October 14-18, 2012 